\title{
Quality Control by Artificial Vision
}

\author{
Edmund Y. Lam \\ University of Hong Kong \\ Department of Electrical and Electronic Engineering \\ Imaging Systems Laboratory \\ Pokfulam, Hong Kong
}

\author{
Shaun S. Gleason \\ Oak Ridge National Laboratory \\ Image Science \& Machine Vision \\ Box 2008, MS 6075 \\ Oak Ridge, Tennessee 37831-6075
}

\author{
Kurt S. Niel \\ Upper Austria University of Applied Sciences \\ Engineering and Environmental Studies \\ Stelzhamerstrasse 23 \\ 4600 Wels, Austria
}

Computational technology has fundamentally changed many aspects of our lives. One clear evidence is the development of artificial-vision systems, which have effectively automated many manual tasks ranging from quality inspection to quantitative assessment. In many cases, these machine-vision systems are even preferred over manual ones due to their repeatability and high precision. Such advantages come from significant research efforts in advancing sensor technology, illumination, computational hardware, and imageprocessing algorithms.

Similar to the Special Section on Quality Control by Artificial Vision published two years ago in Volume 17, Issue 3 of the Journal of Electronic Imaging, the present one invited papers relevant to fundamental technology improvements to foster quality control by artificial vision, and fine-tuned the technology for specific applications. We aim to balance both theoretical and applied work pertinent to this special section theme. Consequently, we have seven high-quality papers resulting from the stringent peer-reviewing process in place at the Journal of Electronic Imaging. Some of the papers contain extended treatment of the authors' work presented at the SPIE Image Processing: Machine Vision Applications conference and the International Conference on Quality Control by Artificial Vision.

On the broad application side, Liu et al. propose an unsupervised texture image segmentation scheme. Using a multilayer data condensation spectral clustering algorithm together with wavelet transform, they demonstrate the effectiveness of their approach on both texture and synthetic aperture radar images. A problem related to image segmentation is image extraction. For this, O'Leary et al. investigate the theory of polynomial moments and show how these moments can be compared to classical filters. They also show how to use the discrete polynomial-basis functions for the extraction of 3-D embossed digits, demonstrating superiority over Fourier-basis functions for this task.

Image registration is another important task for machine vision. Bingham and Arrowood investigate the implementation and results in applying Fourier phase matching for projection registration, with a particular focus on nondestructive testing using computed tomography. Readers interested in enriching their arsenal of image-processing algorithms for machine-vision tasks should find these papers enriching.

Meanwhile, we have four papers dealing with more specific machine-vision tasks. The first one, Yahiaoui et al., is quantitative in nature, using machine vision for real-time passenger counting. Occulsion is a common problem in counting objects and people, and they circumvent this issue with a dense stereovision system, achieving 97 to $99 \%$ accuracy in their tests. On the other hand, the second paper by Oswald-Tranta et al. focuses on thermographic crack detection. An infrared camera is used to detect inhomogeneities, which may indicate surface cracks. They describe the various steps in developing fully automated testing equipment aimed at a high throughput. Another paper describing an inspection system is Molleda et al., which handles flatness inspection of rolled products. They employ optical-laser triangulation and 3-D surface reconstruction for this task, showing how these can be achieved in real time. Last but not least, Presles et al. propose a way to monitor the particle-size distribution of batch crystallization processes. This is achieved through a new in situ imaging probe and image-analysis methods. While it is unlikely any reader may be working on these four specific problems at the same time, we are confident that readers will find these papers inspiring and potentially helpful to their own machine-vision system developments. 
We would like to express our gratitude to Prof. Hamed Sari-Sarraf for spearheading the efforts in organizing this special section, to Prof. Jan Allebach, Editor-in-Chief of the Journal of Electronic Imaging, who afforded us the opportunity, and to the SPIE editorial staff for the wonderful help they have given us over the past one and a half years.

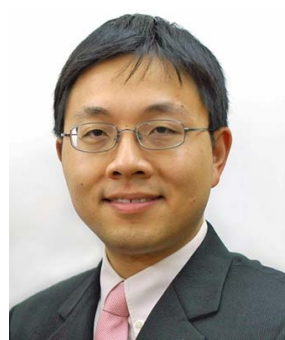

Edmund $Y$. Lam received his BS (with distinction), MS, and $\mathrm{PhD}$ degrees in electrical engineering from Stanford University. $\mathrm{He}$ is now an associate professor in electrical and electronic engineering and a codirector of the computer engineering program at the University of Hong Kong. He is also the founding director of its Imaging Systems Laboratory. His research interests include computational optics and imaging, particularly their applications in semiconductor manufacturing and biomedical systems. Besides his involvement within the university, he is also a topical editor of the Journal of the Optical Society of America $A$ and an associate editor of the IEEE Transactions on Biomedical Circuits and Systems. He is active in conference organizations and currently serves as a program committee member for the SPIE conference on Image Processing: Machine Vision Applications, and OSA's Signal Recovery and Synthe- sis, for which he is the program chair of its 2011 meeting. He is a senior member of IEEE, OSA, and SPIE.

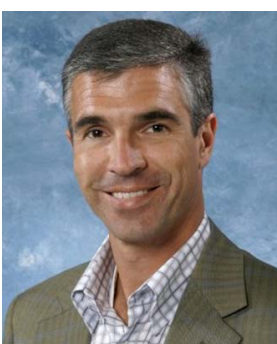

Shaun S. Gleason is group leader of the Image Science and Machine Vision (ISMV) Group at the Oak Ridge National Laboratory (ORNL). He has $20+$ years of experience in applied image processing for in dustrial, security, and medical imaging applications. He earned his BS, MS, and $\mathrm{PhD}$ degrees in electrical engineering from the University of Tennessee, Knoxville, with a research focus on $x$-ray computed tomography and 2-D and 3-D model-based medical image segmentation. In 1998 he cofounded and was the vice president of a preclinical imaging company that successfully transferred ORNL-developed technology into the commercial research sector. He was director of the Preclinical Research Division of Siemens Medical until 2008, when he returned to ORNL to lead the ISMV group. He has authored and coauthored over 100 publications and has been issued 5 patents in the field of computer vision. He is a senior member of the IEEE and an associate editor of the Journal of Electronic Imaging.

Kurt S. Niel: biography and photograph not available. 